\title{
PRIME RINGS WITH INVOLUTION WHOSE SYMMETRIC ZERO-DIVISORS ARE NILPOTENT
}

\author{
P. M. COHN
}

\begin{abstract}
Let $k$ be a field and $R$ the $k$-algebra generated by $x$ and $y$ with the single defining relation $x^{2}=0$. Using free ring techniques we prove that the set of left zero-divisors of $R$ is $R x$. There is a unique involution fixing $x, y$ and this makes $R$ into a prime ring with involution whose symmetric zero-divisors are nilpotent (answering a question by W. S. Martindale). This example also provides us with a subfunctor of the identity whose value is a onesided ideal (answering a question by R. Baer).
\end{abstract}

Recently W. S. Martindale (in conversation) raised the question whether a prime ring with involution exists, not 4-dimensional over its centre, in which every symmetric zero-divisor is nilpotent. He observed that there is a natural candidate over any field $k$, namely the algebra $k\left\langle x, y \mid x^{2}=0\right\rangle$, with involution over $k$ fixing $x$ and $y$, but to verify the desired property seems not entirely trivial. The proof given here ${ }^{1}$ illustrates the use of free ring techniques described in [1]. In fact we shall prove the

THEOREM. Let $R$ be an algebra over a field $k$, generated by $x, y$ with the single defining relation $x^{2}=0$; then any left zero-divisor is in $R x$.

Proof. Let $F=k\langle x, y\rangle$ be the free $k$-algebra on $x$ and $y$, and let $a$ be the ideal of $F$ generated by $x^{2}$; then the monomials which do not have $x^{2}$ as factor are $k$-linearly independent $\bmod a$. Given $a, b \in F$ such that $a, b \notin \mathfrak{a}$ but $a b \in \mathfrak{a}$, it follows that neither $a$ nor $b$ has a constant term. Write $a=a_{0}+a_{1}, b=b_{0}+b_{1}$, where $a_{0}, a_{1}$ end in $x, y$ respectively and $b_{0}, b_{1}$ begin with $x, y$ respectively. By omitting terms we may assume that neither $a$ nor $b$ contains a monomial with $x^{2}$ as factor, and since the others are linearly independent $\bmod a$, we have $a_{0} b_{1}+a_{1} b_{0}+a_{1} b_{1}=0$, i.e.

$$
a b=a_{0} b_{0} \text { in } F \text {. }
$$

Received by the editors October 19, 1972.

AMS (MOS) subject classifications (1970). Primary 16A12; Secondary 16A28, 16A34, 16A06.

Key words and phrases. Prime ring, involution, zero-divisor, nilpotent, free ring, weak algorithm.

${ }_{1}^{1}$ Professor Martindale informs me that Estes has also obtained a proof. Another proof, using coproducts, has been found by G. M. Bergman.

(c) American Mathematical Society 1973 
This equation shows that $a_{1}=0$ if and only if $b_{1}=0$, i.e. $a \in F x$ iff $b \in x F$. To complete the proof we use induction on the number of factors of $a$. If $a$ is an atom (i.e. unfactorable) then since $a, a_{0}$ have no constant term, their highest common left factor is a nonunit, and is therefore $a$, so $a_{0}=a c$. By comparing degrees we see that $c$ is a unit, so $a=c^{-1} a_{0} \in F x$ as claimed. Next let $a=a^{\prime} p$, where $p$ is an atom, then $p \notin \mathfrak{a}$; if $p b \in \mathfrak{a}, p \in F x$ and so $a \in F x$. Hence we may assume that $p b \notin \mathfrak{a}$, but $a^{\prime} p b=a b \in \mathfrak{a}$, so by the induction hypothesis, $a^{\prime}=a^{\prime \prime} x, p b=x c$, and it follows by the weak algorithm [1, Chapter 2] that $p=x q+\lambda$, where $\lambda \in k$. If $\lambda=0, p$ is associated to $x$ and the result follows, so assume $\lambda \neq 0$. Then $x c=(x q+\lambda) b$, hence $\lambda b=x(c-q b)$, so $b \in x F$, and therefore $a \in F x$, as we wished to show.

From the theorem it is clear that $R$ is prime: if $a, b \neq 0$, then $a y b \neq 0$, so $a R b \neq 0$, and of course $R$ is infinite dimensional and central over $k$. Moreover, we have the

COROLlaRY. If in $R$ we take the involution fixing $x, y$, then every symmetric zero-divisor is nilpotent.

For if $c$ is a symmetric zero-divisor, say $c d=0$, then $c=a x$, and by symmetry, $c=x a^{*}$, hence $c^{2}=a x^{2} a^{*}=0$.

The above theorem can also be used to solve the following problem, raised by R. Baer: Find a ring $R$ with a left ideal invariant under all automorphisms of $R$, yet not two-sided. The ring $R$ of the theorem and $R x$ clearly form such a pair. More generally, Baer asks for a subfunctor of the identity whose value is a left ideal which is not always two-sided. The first examples that come to mind are one-sided functors (i.e. not invariant under passage to the opposite ring), but their values are usually two-sided ideals, e.g. the left socle. An example meeting the above requirements is "the left ideal generated by all the nilpotent elements". This is easily verified to be a subfunctor of the identity and, in the ring $R$ of the theorem, its value is $R x$.

\section{REFERENCE}

1. P. M. Cohn, Free rings and their relations, Academic Press, New York, 1971.

Department of Mathematics, Bedford College, Regent's Park, London, NW1 4NS, ENGLAND 